\title{
Robusta : un satellite pédagogique
}

\author{
Sylvie Jarrix, Gérard Gervois, Jérôme Boch, Jean-Roch Vaillé, Philippe Nouvel, Annick Penarier, \\ Laurent Dusseau \\ prenom.nom@ies.univ-montp2.fr \\ Institut d'Electronique du Sud - Université Montpellier 2 \\ UMR UM 2 - CNRS 5214 \\ Place Eugène Bataillon \\ 34095 Montpellier cedex 5 - FRANCE
}

\begin{abstract}
RESUME : Robusta est un satellite de norme cubesat entièrement conçu et réalisé par les étudiants issus de formations diverses de l’Université Montpellier 2 : IUT , école d'ingénieur, licence et master EEA. C'est un projet en partenariat avec le CNES et soutenu par d'autres grands industriels du secteur électronique. Le satellite a pour mission scientifique de mesurer la dégradation sous l'effet de rayonnements ionisants de composants électroniques basés sur des transistors bipolaires. Ces dégradations seront par la suite comparées à celles obtenues par des méthodes de test au sol. Ce projet est une expérience de nature industrielle qui reste de par sa durée, son coût et sa technicité à l'échelle des étudiants. Robusta, en tant que système, permet aux étudiants de niveau bac +2 à bac +8 de développer des prototypes techniques pointus et d'améliorer leur sens de la communication tout en découvrant le monde du spatial.
\end{abstract}

Mots clés : dispositif pédagogique, aéronautique et spatial, étude système

\section{INTRODUCTION}

Les satellites sont un vecteur exceptionnel d'apprentissage de la conception de systèmes interdisciplinaires et de leur intégration. Le projet de "cubesat" a été conçu par le professeur R. Twiggs ${ }^{[1]}$ dans les années 2000 aux Etats-Unis, et mis en place à l'université polytechnique de Californie (Calpoly). R. Twiggs a montré qu'un tout petit satellite pourvu d'une vraie mission, de par sa relative simplicité et faible coût par rapport aux gros systèmes spatiaux, peut convenir à un programme d'enseignement complet. Depuis, plus d'une trentaine d'universités dans le monde ${ }^{[2][3][4][5][6][7]}$ se sont lancés dans ce type de projet pédagogique, dont maintenant l'Université Montpellier 2 avec Robusta (Radiation On Bipolar University Satellite Test Applications). L'objectif est de former des étudiants par l'expérience en conditions réelles de la conception d'un système. Ils doivent s'impliquer depuis la définition de la mission jusqu'à l'exploitation des données de mesure, en passant par toutes les phases de conception, approvisionnement des composants, réalisation de prototypes et tests.

\section{CONTEXTE}

En 2000, Alcatel-Space achetait un cubesat, SACRED (Space And Cosmic Rays Radiation on Electronic Devices) à l'Université d'Arizona afin d'étudier l'effet des radiations ionisantes sur des composants MOS de puissance. La charge utile était entièrement conçue par les étudiants de l'Université Montpellier $2^{[8]}$ sous l'encadrement d'enseignants-chercheurs de l'IES (Institut d'Electronique du Sud). L'échec du lanceur DNEPR-1 entraîna la perte du picosatellite. Néanmoins SACRED, en dévoilant toutes les parties constitutives d'un tel satellite, mit en évidence le fait que l'Université Montpellier 2 possédait au sein de ses filières et de ses laboratoires de recherche toutes les compétences requises pour mener un tel projet à terme, et ce dans toutes les phases de conception.

En 2006 le CNES (Centre National d'Etudes Spatiales) lançait un appel à idées à destination des étudiants dans le cadre des projets EXPRESSO (EXpérimentations et PRojets Etudiants dans le domaine deS Systèmes Orbitaux et ballons stratosphériques). Sur huit réponses obtenues trois ont été retenues : le projet PLAGE : PLAteforme GEnérique d'étude de la micro-gravité de l'ENSEIHT-Toulouse, PARAPOM (PARAchute for Particule Occurrence Monitoring) : mesure de densité de particules sur ballon stratosphérique par l'INSAToulouse et enfin le projet Robusta de l'Université Montpellier 2 présenté par son porteur $M$. le professeur Laurent Dusseau : un pico-satellite présentant une véritable expérience scientifique.

http://www.cnes.fr/web/CNES-fr/4175-expresso.php

Le projet Robusta consiste en la conception puis la réalisation d'un pico-satellite et ce, entièrement par des étudiants. Jusqu'à présent environ 120 étudiants de niveau licence, licence professionnelle, master et doctorat, ont participé au projet.

Plusieurs parties peuvent être dégagées de ce projet : l'étude système et la gestion de projet associée, la structure mécanique, les parties électroniques relatives à plusieurs domaines de l'EEA : la gestion de l'énergie, la charge utile, la carte contrôleur, la carte radiocommunication, la station sol associée. Il faut enfin rajouter une partie communication et vulgarisation auprès des médias. Le satellite doit être pensé, réalisé et testé par les étudiants, guidés par des enseignants experts dans le domaine concerné. Ils sont également chargés de la gestion de projet.

Voir la structure du satellite et les formations impliquées ici:sur vidéo 1:

http://www.ies.univ-montp2.fr/videorobusta/ 


\section{PRESENTATION DU SATELLITE ET DE SA MISSION}

Le satellite répond aux normes cubesat : c'est un cube de $10 \mathrm{~cm}$ de côté (cf. structure métallique figure 1), de poids $1 \mathrm{~kg}$, de consommation maximale $1 \mathrm{~W}$.. Pour les besoins spécifiques de la mission un "trou" dans le blindage est pratiqué, assurant ainsi une exposition directe aux radiations des composants sous test.

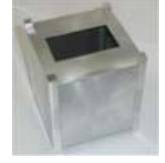

fig. 1 : prototype de la structure métallique

Sa conception, sa réalisation et les tests sont prévus sur une période de trois ans. Le choix du lanceur et la date de lancement ne sont pas encore fixés. L'équipe de Robusta a participé en janvier 2008 au Vega Maiden Flight Workshop à Noordwijk (Pays-Bas) à l'ESA (Agence Spatiale Européenne) afin de présenter le projet $^{[9][10]}$.

Elle a ainsi concouru parmi une trentaine d'universités européennes pour une place sur le vol de qualification de la nouvelle fusée européenne Vega. Le résultat du concours n'est pas encore dévoilé, néanmoins le projet semble avoir fait forte impression.

http://www.esa.int/esaCP/index.html

Ce satellite a une véritable mission scientifique : mesurer la dégradation due aux rayonnements ionisants de composants électroniques basés sur des transistors bipolaires. Les composants sous test choisis sont les comparateurs de tension LM139 et les amplificateurs de tension LM124, composants souvent utilisés à bord d'engins de vol. Ces dégradations sont mesurées par l'intermédiaire de mesures de courants, de tensions, de température et de dose reçue. Cette dose correspond à un dépôt d'énergie par unité de masse. Les résultats seront ensuite comparés à ceux obtenus par une méthode de test au sol mise au point par des chercheurs du laboratoire universitaire IES de Montpellier ${ }^{[11]}$.

http://www.ies.univ-montp2.fr/

La mesure de dose, soit le dépôt d'énergie par unité de masse, est effectuée grâce à un capteur OSL également mis en œuvre au laboratoire ${ }^{[12]}$.

La durée de la mission en vol est fixée à trois ans. Les données seront mesurées au minimum toutes les douze heures. Elles seront ensuite transmises à la station sol du campus de Montpellier en utilisant des fréquences et un protocole radio-amateur. La méthode de transmission se fera en broadcast, c'est-à-dire en continu toutes les trois minutes, que le satellite soit dans une fenêtre de visibilité ou non. Un partenariat avec l'université de Würzburg en Allemagne et l'université d'Arizona aux Etats-Unis permettra d'utiliser leurs stations sol également pour récupérer les données. La déclaration officielle des fréquences utilisées est en cours. Pour la station sol, l'autorisation de poser des antennes sur le toit d'un bâtiment du campus de Montpellier 2 est accordée.

Enfin l'alimentation du satellite se fera par l'intermédiaire de batteries Saft Li-Ion, qui se rechargeront par les cellules solaires spécifiques spatiales Azurspace.

On remarque ici déjà trois points forts. D’abord, la durée de conception et réalisation. Celle-ci est suffisamment courte par rapport à un gros satellite pour que les étudiants aient vraiment le sentiment d'en avoir réalisé une partie importante au cours d'une seule année universitaire. Certains étudiants, qui auront démarré un projet sur Robusta lors de leur année de licence, pourront même y participer jusqu'au lancement. Le deuxième point fort est l'intérêt proprement scientifique de la mission. Ceci est une plus value pour le satellite en lui-même, mais est surtout un catalyseur pour la motivation des étudiants qui ont le sentiment de fabriquer quelque chose d'utile. Ceci peut les attirer vers des études longues, et d'ailleurs, le projet se déroulant pour beaucoup en laboratoire universitaire ils expriment déjà en licence troisième année ou master première année le désir de clore leurs études par une thèse. Par l'intérêt qu'il suscite parmi les industriels de l'électronique, des échanges avec des industriels se forment et des propositions de stage en industries s'ensuivent. Enfin, de par le fait que ce satellite doit être réellement mis en orbite, le succès de la mission dépend directement des étudiants ce qui a pour effet de les responsabiliser très tôt.

\section{UN PROJET FEDERATEUR, POLYNI- VEAUX, POLY SITES, POLY FORMATIONS}

L’IUT de Nîmes avec les sections GMP (Génie Mécanique et Productique), GEII (Génie Electrique et Informatique Industrielle) l'école Polytech-Montpellier avec la section ERII (Electronique Robotique et Informatique Industrielle) et enfin la licence et le master EEA de l'Université Montpellier 2 sont impliquées. http://www.univ-montp2.fr/

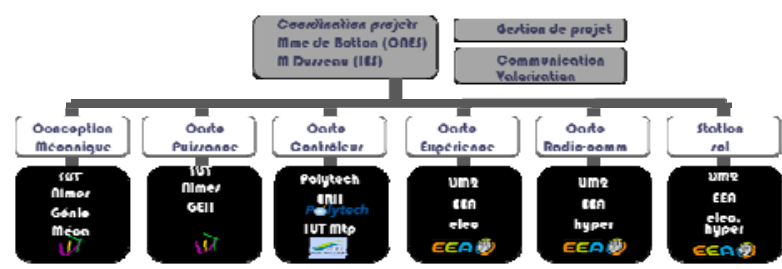

fig. 2 : Organigramme des formations impliquées

Les spécificités de chaque formation sont mises à contribution : la structure mécanique, le dimensionnement, le positionnement des cartes électroniques, et les problématiques du système de lancement par l'intermédiaire d'un p-pod sont pris en charge par la section GMP de l'IUT de Nîmes. Le sous-système carte alimentation, la gestion de l'énergie des batteries et des cellules solaires est assurée par la section GEII de 
l'IUT de Nîmes. Le sous-système carte contrôleur qui gère les ordres entre cartes et stocke les données de mesure est mis en œuvre par la section ERII de Polytech Montpellier. Le sous-système carte expérience qui comprends les composants sous test, le capteur de dose, les capteurs de température est conçu par la formation EEA de l'université, plus particulièrement les parcours Electronique - fiabilité et Capteurs. Les soussystèmes carte radiocommunication et station sol sont élaborées par la formation EEA, plus particulièrement le parcours Hyperfréquences. Cette répartition est représentée sur l'organigramme de la figure 2.

Les étudiants sont impliqués par l'intermédiaire de projets ou stages intégrés à leur cursus. Les sujets d'études sont donc adaptés à la durée et au niveau du stage requis pour la validation d'un diplôme. Ainsi une partie d'un sous-système pourra être étudié sur une trentaine d'heures pour des étudiants de licence première année, ou faire l'objet d'un stage de cinq mois pour les masters deuxième année. Au niveau du doctorat, la partie mission scientifique ayant pour but une validation d'une méthode de test sur des composants particuliers est bien entendu privilégiée. La figure 3 représente la proportion d'étudiants impliqués par niveaux.

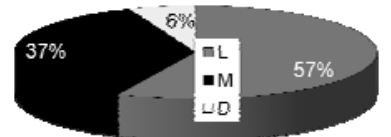

fig. 3 : répartition en pourcentage des étudiants de Robusta selon leur cursus $L$ : licence, $M$ : master, $D$ : doctorat

Parmi ces étudiants, certains choisissent, en plus de l'étude d'un sous-système technique, de s'occuper de la coordination complète du projet. Il leur revient de provoquer les réunions, d'écrire et de faire valider les compte-rendus, de s'assurer de la cohérence des solutions retenues. Ils ont alors à fournir un travail de coordination et de communication important.

On peut ici faire valoir quatre points forts du projet : autour de Robusta sont proposés des stages ou projets de niveau industriel, adaptés au niveau de l'étudiant, pris en compte dans leur cursus, et permettant un brassage sur un même point technique d'étudiants de niveaux différents. Par ailleurs, ce projet d'envergure a suscité l'engouement de certains étudiants en situation d'échec. Ils ont directement vu l'application de leurs cours et la possibilité d'un travail sur un projet concret industriel. Ils se sont alors remotivés par ce biais et sont revenus dans le cursus universitaire.

\section{UN PROJET TECHNIQUE POINTU}

\section{Déroulement du projet}

L’objectif pédagogique du projet est complet : pour chaque partie un cahier des charges est établi. Ensuite il s'agit de mener une recherche bibliographique sur les systèmes déjà, ou susceptibles d'être, employés sur ce type de satellite. Cette étude bibliographique est systé- matiquement suivie d'un choix argumenté des solutions. Enfin la réalisation proprement dite de prototypes et de leurs tests est induite. Chaque étape de choix, de réalisation et de test fait l'objet d'un compterendu. Les étudiants apprennent ainsi, et ce quel que soit la durée de leur stage ou leur niveau, à effectuer une recherche bibliographique en tenant compte des contraintes techniques, et à mettre en application leur savoir faire pour la réalisation et les tests. Les étudiants doivent par ailleurs dépasser les connaissances théoriques acquises en cours. Ils doivent ainsi effectuer une synthèse de plusieurs domaines pour pouvoir réaliser des prototypes fonctionnels, depuis le routage de circuits jusqu'à l'implantation de certaines connectique, ce qui relève tout à fait d'un programme d'ingéniorat.

\section{Mise en commun d'un savoir-faire}

Une plateforme de savoir commun se met en place pour ce qui est des composants électroniques. Ainsi l'environnement radiatif spatial, au cœur de la mission de Robusta, doit être parfaitement intégré et compris par tous les acteurs du projet. En effet les composants choisis pour les sous-systèmes autres que ceux sous tests doivent pouvoir résister un minimum aux radiations. Les étudiants sont donc tous sensibilisés au problème spatial, au choix de composants et aux problèmes de blindage et de durcissement système.

La nécessité de compacter au plus le système et d'avoir une alimentation restreinte influe également pour tous sur le choix des composants.

Enfin l'approvisionnement des composants n'est jamais simple et les étudiants ont l'occasion de le découvrir en temps réel. Tout d'abord ils se familiarisent avec les fondeurs ou distributeurs spécifiques ou encore les logiciels spécifiques à leur domaine, par exemple OMERE pour le calcul de doses reçues, ou les fournisseurs mini-circuits ou Hittite pour les composants radiofréquence. En même temps ils sont sensibilisés à l'environnement en découvrant et choisissant de préférence des composants RoHS (Restriction of the use of certain Hazardous Substances). Ils peuvent également directement aborder des technologies avancées comme les composants CMS (Composants Montés en Surface) et les circuits imprimés double face pour répondre aux contraintes de poids et de taille. Il est à noter que dans les projets souvent proposés à l'université ces contraintes sont rarement prises en compte.

Des industriels (Intersil, Texas Instrument) fort intéressés par la mission de Robusta se sont proposés pour fournir les composants requis, dans la limite des disponibilités. Les étudiants doivent ainsi suivre une procédure particulière : définir leurs besoins, repérer les composants adéquats, établir une liste complète et exhaustive des composants et de leurs références aux fabricants ou distributeurs concernés. Dans la mesure où certains composants ne peuvent être fournis par les industriels sponsorisant le projet, ils se doivent de trouver au plus vite des équivalents. Si cette dernière 
option s'avère infructueuse les étudiants sont soumis aux contraintes de délais comme pour tout autre projet industriel d'envergure ainsi qu'aux contraintes de nombre, et donc de prix, dans le cas de composants très spécifiques.

\section{Exemples d'applications}

L'exemple du sous-système carte contrôleur est tout à fait intéressante. Cette carte doit gérer l'ordonnancement du satellite. Sa conception est plus particulièrement dévolue à la section électronique de Polytech Montpellier. Au cours de réunions inter-soussystèmes, il a été choisi d'utiliser un bus CAN pour la communication entre les différents sous-systèmes. Par rapport au bus I2C, le bus CAN semblait présenter davantage de robustesse. Or à ce jour, le programme pédagogique ne prévoyait d'enseigner que l'étude et la programmation du bus I2C. Les étudiants ont ainsi pu acquérir un savoir complémentaire. La transmission du savoir qui était jusque là unidirectionnelle, c'est-à-dire de l'enseignant vers l'étudiant, s'est progressivement transformée en transmission bidirectionnelle lorsque les encadrants ont dû valider et conseiller les réalisations. D'autre part les étudiants ont pu, lors de la conception du prototype, développer leur propre protocole d’échanges de données.

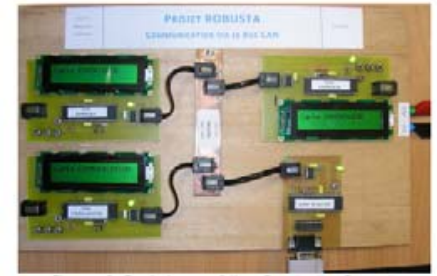

fig. 4 : prototype du sous-sensemble carte contrôleur.

Ils ont fabriqué un prototype (cf. Fig 4) sur lequel les quatre sous-systèmes électroniques de Robusta ont été émulés. Ils ont également implantés un "sniffer" pour les tests et vu en situation réelle son activité. Ce prototype est actuellement en phase de test aux radiations, sujet nouveau pour de nombreux étudiants.

Pour le sous-système radiocommunication, les étudiants ont compris le sens de bande de fréquences allouées pour une application. Apres avoir étudié diverses architectures d'émission-réception ils ont opté pour un système à deux fréquences séparées : $345,25 \mathrm{MHz}$ pour l'émission et $145,95 \mathrm{MHz}$ pour la réception. Le choix des composants et notamment des amplificateurs se sont fait directement en concertation avec les étudiants du sous-système station sol en fonction du budget de liaison. Néanmoins, en plus d'une culture nécessaire sur les problématiques propres aux radiofréquences, ils ont dû mettre en œuvre des procédés de traitement du signal lors du choix du type de modulation et démodulation. La simulation n'a pas été oubliée, notamment pour les antennes du satellite (cf. figure 5) qui ont été entièrement simulées à l'aide d'un logiciel professionnel spécifique hyperfréquences CST Microwave Studio.

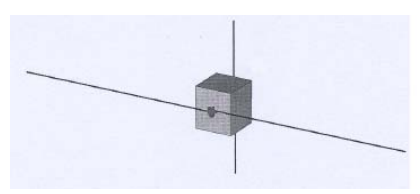

fig. 5 :modèle de la structure cubesat avec ses antennes sous simulateur CST MS pour optimisation du comportement des antennes.

Pour la station sol, par souci pédagogique, il a été demandé aux étudiants de réaliser un système d'émission-réception. Celui-ci par sécurité sera doublé par un système commercial radioamateur. Néanmoins, là encore, les étudiants ont été impliqués puisque ce sont eux qui ont fait le choix du système à acquérir. Ils ont également effectué de nombreuses recherches pour le choix des antennes des stations sol.

Pour la carte expérience, du design de circuit électronique a dû être amplement conçu et testé. En effet, chaque circuit intégré sous test (LM124 et LM139) comporte huit éléments sur lesquels il faut mesurer des courants, des tensions, des températures et des doses. Il a donc fallu trouver une architecture à base de commutateurs analogiques pilotés par le microcontrôleur pour multiplexer les mesures prises sur les différentes pattes des composants. Les étudiants ont dû choisir un BUS permettant de gérer le grand nombre d'adresses allouées aux interrupteurs et ainsi découvrir les bus I2C et SPI.

Ces exemples ne sont pas exhaustifs et pour chaque sous-système de telles particularités peuvent être mises en exergue.

Au travers de ces exemples, on peut mettre en avant un autre point fort du projet Robusta : c'est l'acquisition d'un savoir poussé et complémentaire des seules formations théoriques en conception de système dans son environnement.

\section{UN PROJET DE COMMUNICATION}

Le projet Robusta regroupant diverses formations sur Nîmes et Montpellier, la communication est essentielle pour l'avancement du projet et surtout une parfaite cohésion entre les différentes parties du satellite. Les étudiants participent régulièrement aux réunions provoquées à l'intérieur des sous-systèmes mais surtout aux réunions inter-sous-systèmes. Avant chaque réunion, celle-ci devant être préparée, ils doivent effectuer une synthèse de leurs résultats. Les discussions qui suivent les présentations de résultats ou de choix de solutions doivent être comprises par tous, même les non spécialistes du domaine. Ainsi les étudiants font tout naturellement un effort pour avoir une vision large $d u$ projet. Ils sont pointus sur leur sujet de stage propre, mais acquièrent rapidement et de façon autonome des connaissances de base dans d'autres domaines. Ils établissent donc leurs solutions en tenant compte des problématiques des autres sous-systèmes, sans quoi le satellite complet en tant que système ne pourrait pas fonctionner. 
Les comptes-rendus suivent un guide normatif édité par le CNES. Les étudiants doivent donc se conformer à ces guides, tout comme ils le feraient dans une entreprise. Chaque étape de conception et de choix est largement détaillée en faisant la part entre solutions techniques, les problématiques d'entrées-sorties des soussystèmes, les implications sur l'environnement, en particulier pour respecter la charte des débris spatiaux.

Par ailleurs en février 2008 une exposition a été montée sur le campus de l'université autour du projet Robusta. Les étudiants qui le souhaitaient avaient l'opportunité soit de présenter un poster sur le sous-système sur lesquels ils travaillent, soit de faire une présentation orale. Tous les sous-systèmes du satellite, structure mécanique et station sol compris, ont fait l'objet d'une communication par ces deux moyens. Un gros travail a été fourni par les étudiants, principalement de master première année, pour faire passer un véritable message scientifique par l'image. Le type de communication par poster, si prisée au sein de la communauté scientifique, n'est quasiment pas enseignée et demande un effort considérable de synthèse pour garder un fond scientifique vrai, tout en lui conférant un aspect non rébarbatif.

Ensuite les étudiants ont présenté en binôme quinze minutes d'exposé sur l'étude d'un sous-système particulier à l'aide d'un support type power point. Ils ont dû là encore s'attacher à une synthèse claire de leur travail pour mettre en exergue les choix importants et l'avancement réel du satellite. Ils ont pu également par ce biais prendre la parole avec un micro dans une vraie salle de conférences, et ce devant un public cosmopolite composé de camarades, enseignants et public adulte passionné de sciences spatiales. Les étudiants managers tenaient le rôle de chairman. Vu le nombre élevé de présentations attendues, les délais ont été scrupuleusement respectés.

Voir des extraits de l'exposition ici:vidéo 2:

http://www.ies.univ-montp2.fr/videorobusta/

Cet exercice a été largement profitable ainsi que le corps enseignant a pu le constater par la suite. En effet ces mêmes étudiants ont dû présenter oralement leurs travaux lors de soutenances de projets. Le niveau des présentations en licence troisième année et master première année ont largement dépassé le niveau moyen habituel.

On remarque ainsi encore un point fort du projet Robusta : il permet aux étudiants d'apprendre à communiquer des résultats techniques, acquérir un savoirfaire en matière de présentation des résultats scientifiques et de prise de parole.

\section{UN PROJET MEDIATISE}

Devant l'originalité de ce projet fédérateur, les étudiants ont été largement sollicités par les journalistes pour des reportages télévisés ou des articles dans les journaux régionaux ${ }^{[13][14][15][16]}$. Cette médiatisation a eu le mérite de faire connaître le projet sur la région Languedoc Roussillon auprès notamment des jeunes. Cette façon ludique de présenter les sciences a eu pour conséquence de susciter l'intérêt de nombre de lycéens et autres jeunes, susceptibles d'être par la suite attirés vers les diverses formations universitaires. Ceci est un atout non négligeable quand on connaît la baisse de fréquentation pour les filières scientifiques.

Par ailleurs les étudiants impliqués ont dû adapter leur langage technique au tout public et s'essayer à la vulgarisation scientifique.

\section{UN PROJET DE PARTENAIRES}

\section{Un projet peu coûteux}

Le coût total du satellite, avec la construction d'une station sol et le déplacement d'un noyau dur d'étudiants pour assister au lancement, a été estimé à $90 \mathrm{k} €$. Les formations universitaires en premier lieu couvrent plus de $25 \%$ du coût total. En effet pour chaque formation des stages pratiques sont obligatoires pour la validation du diplôme et des ressources y sont allouées. Le partenaire CNES contribue directement en finançant chaque phase du projet. Un financement est prévu de la part des institutions locales : université, laboratoire de recherche, Région Languedoc-Roussillon.

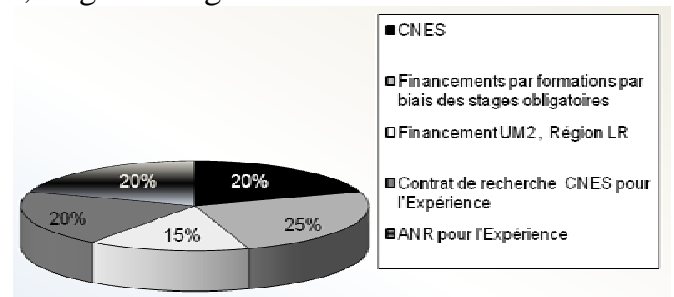

fig. 6 : répartition en pourcentage des différentes sources de financement

Une source de financement importante provient de contrats de recherche sur la charge utile et la validation de la méthode de test au sol. La contribution de chacune des sources de financement est représentée figure 6 .

\section{Un projet sponsorisé}

De nombreux industriels parrainent le projet. En effet, pour eux, Robusta est avant tout un excellent vecteur de communication grand public. Ils sont également intéressés pour embaucher des étudiants en Sciences pour l'Ingénieur bien formés dans le domaine. Pour certains, directement impliqués dans l'électronique spatiale, il s'agit de supporter la mise au point de la méthode de qualification de leurs composants. C'est le cas de la société Intersil qui ainsi fournit des composants durcis. Texas Instrument (TI) également fournit des composants commerciaux et en outre, met à disposition une dizaine de kits de développement de Microcontrôleurs. La société TRAD, implantée à Labège, a fourni plusieurs licences de leur logiciel FASTRAD 
pour modéliser le satellite et calculer la dose reçue par chaque composant. Un exemple est donné fig 7.

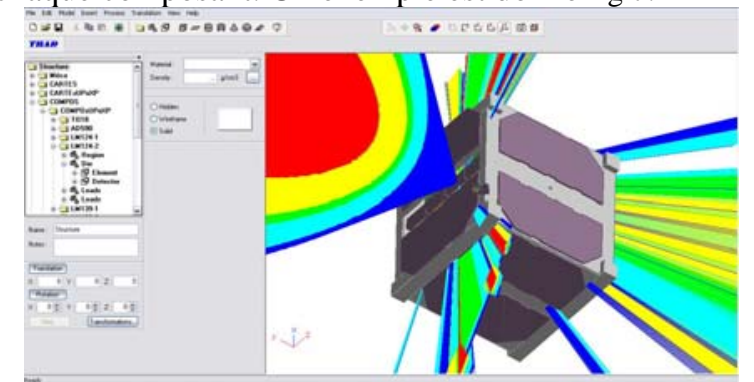

fig 7 :exemple de modélisation pour le calcul de la dose $a b-$ sorbée par un composant de Robusta avec le logiciel FASTRAD.

La société SAFT fournit elle les batteries Li-Ion, en échange de données sur leur comportement en vol. Des mesures de suivi de batteries seront donc également effectuées au cours de la mission. Enfin, le groupe Cofidur s'est engagé à fournir le modèle de vol des cartes et ce, en associant les étudiants concepteurs qui pourront assister en direct à la naissance du prototype.

Ainsi, il apparaît qu'un projet interdisciplinaire d'envergure peut tout à fait être mené au sein d'une université. La condition toutefois est de présenter un intérêt scientifique majeur pour la communauté scientifique et les industriels afin d'obtenir des sources de financement complémentaires.

\section{CONCLUSION}

Le projet Robusta vise à concevoir un pico-satellite ayant pour mission de mesurer les dégradations encourues par des composants bipolaires sous rayonnement ionisant. Les mesures, sur trois ans, seront comparées à celles obtenues au sol par une méthode de test. Sous l'encadrement d'enseignants du supérieur et d'enseignants chercheurs, quelque 120 étudiants de l'université Montpellier 2, depuis la licence professionnelle d'IUT, les licence et master EEA, et le doctorat, se sont impliqués dans le projet par des projets ou stages intégrés à leurs cursus. En plus de la culture spatiale nécessaire, différents domaines techniques sont abordés, depuis la gestion de l'énergie aux radiocommunications ou encore la programmation de micro-contrôleurs. L'intérêt d'un tel projet, à vocation scientifique réelle, est de permettre aux étudiants de réaliser des prototypes, d'acquérir une vision système, et de communiquer sur le projet de différentes manières. Même si le lancement tant attendu du satellite Robusta devait tourner à l'échec, d'un point de vue pédagogique Robusta est déjà un succès.

\section{REMERCIEMENTS}

Ce projet est soutenu par le CNES. Nous tenons en particulier à remercier Mme Laurence de Botton, qui en tant que coordinatrice du projet pour le CNES le suit activement et régulièrement.
Nous remercions les sociétés Trad, Intersil, Texas Instrument, Saft et Cofidur qui sponsorisent le projet Robusta.

L'équipe Robusta remercie également $M$ Eric Picard de l'IES pour toute l'aide apportée sur le traitement et la diffusion des fichiers numériques.

\section{Bibliographie}

[1] H. Heidt, J. Puig-Suari, A. Moore, S. Nakasura, R. Twiggs, "Cubesat : a new generation of picosatellite for education and industry low-cost space experimentation", Proc. $13^{\text {th }}$ Annual AIAA/USU small satellite Conf., Logan, UT, Août 2000.

[2] Cubesat Suisse : (consortium écoles suisses romandes dont l'Ecole Polytechnique Fédérale de Lausanne) : http://swisscube.epfl.ch/.

[3] Cubesat Danois: Université d'Aalborg : http://www.cubesat.auc.dk/.

[4] Cubesat de California Polytechnic State University : http://polysat.calpoly.edu/.

[5] Cubesat Italien : S. Kurnaz, F. Ince, S. Onbasiouglu (Eds.) "AtmoCube : Observation of the Earth Atmosphere from the Space to Study" Proc. of Intern. Conf. on "Recent Advances in Space Technologies", IEEE 03EX743, ISBN: 0-78038142-4, p. 179-184, 2003.

[6] Cubesat Hawaiien : W.A. Shiroma, A. T. Ohta, M. A. Tamamoto, "The University of Hawaii CubeSat: a multidisciplinary undergraduate engineering project" 33rd Annual Conf. Frontiers in Education, Nov. 2003, ISBN: 0-7803-7961-6.

[7] Cubesat Japonais : Y.Tsuda, N.Sako, T.Eishima, T.Ito, Y.Arikawa, N.Miyamura, K.Kanairo, S.Ukawa, S.Ogasawara, S.Ishikawa, S.Nakasuka, "University of Tokyo's CubeSat "XI" as a Student-Built Educational Pico-Satellite -Final Design and Operation Plan-", 23rd Inter. Symposium of Space Technology and Science, Matsue, 2002.

[8] L. Dusseau, J. R. Vaillé, F. Saigné, J. Gasiot, L. Cresciucci, D. Campillo, C. Chantry, U. Fink, J. Grunenfelder, P. Calvel, J. D. Gayrard, "Cubesat SACRED : a student project to investigate radiation effects", Proc. $8^{\text {th }}$ IEEE Radecs Conf. 2005.

[9]http://www.esa.int/esaED/SEMSJ8QR4CF_index_0 .html

[10] L. Dusseau, Proposal to Vega Maiden Flight Payload, Vega Maiden Flight Workshop, 23-25 Janvier 2008.

[11] J. Boch, "Estimation of Low Dose Rate Degradation on Bipolar Linear Integrated Circuits Using Switching Experiments", IEEE Trans. Nuclear Science, vol. 52, p.2626-2621, decembre. 2005.

[12] J. R. Vaille, F. Ravotti, P. Garcia, M. Glaser, S. Matias, K. Idri, J. Boch, E. Lorfevre, P. J. McNulty, F. Saigne, L. Dusseau, "Online dosimetry based on optically stimulated lumines- 
cence materials" IEEE Trans. on Nuclear Science, vol. 52, Issue 6, Decembre 2005 pp. 2578 - 2582.

[13] "Les étudiants ont défendu Robusta à l'ESA", Midi-Libre Montpellier, 31 Janvier 2008.

[14] "Le satellite étudiant Robusta est sur le pas de tir", Midi-Libre Montpellier, 21 Janvier 2008.

[15] "Un satellite construit par des étudiants bientôt en orbite", Le Figaro, 27 décembre 2007.

[16] "Un satellite construit par des étudiants" Vivre en Languedoc-Roussillon, décembre 2007. 